\title{
Large-Scale Patterns of Prominences in the Global Solar Cycles During 1880-1995
}

\author{
Dirk K. Callebaut \\ Physics Dept. UIA, University of Antwerp, B-2610 Antwerp, Belgium \\ Valentine I. Makarov and Ksenia S. Tavastsherna \\ Pulkovo Astronomical Observatory, 196140, St. Petersburg, Russia
}

\begin{abstract}
The zonal distribution of prominences, their poleward migration from the sunspot zone to the poles, the polar magnetic field reversals and a correlation of the mean latitude of filament bands at minimum activity with the maximum of Wolf number in the next cycle are briefly discussed for the period 1880-1995. The need for research on the longterm latitude distribution of the prominences is emphasized. New results concerning long-term variations of the torsional oscillations of the Sun and quasi-periodic oscillations of the latitude zonal boundaries from an analysis of $\mathrm{H}_{\alpha}$ charts (1915-1990) are given.
\end{abstract}

\section{Introduction}

Prominences are one of the main manifestations of solar activity. Moreover they have the advantage of occurring at all latitudes from the poles to the equator. The latitude-time distribution of prominences is not random. They show the pattern and evolution of the zonal structure of the large-scale magnetic field and demonstrate the process of polar magnetic field reversal. Flares and coronal mass ejections are certainly linked with the instability of prominences. The main observational features concerning the large-scale pattern of the prominences are derived using observational data for 11 cycles of solar activity (Makarov and Sivaraman 1989, Callebaut and Makarov 1992, Makarov 1994).

It has been shown that there is a zonal structure in the distribution of the prominences (Makarov 1984, Makarov and Mikhailutsa 1992). In each hemisphere the predominent distribution of prominences in the polar, middle and equatorial zones may be detected. These zones coincide with the boundaries of the large-scale rotational symmetric component of the magnetic field.

No phase of the cycle shows a regular equatorward migration of the prominence zonal boundaries or of the single filaments. However, they show a poleward migration with a velocity varying from $5 \mathrm{~ms}^{-1}$ to $40 \mathrm{~ms}^{-1}$ starting after minimum activity up to the polar magnetic field reversal (Makarov 1984, Callebaut and Makarov 1992).

The trajectories of the boundaries of magnetic regions of one polarity were investigated as they migrate to high latitude with the progress of solar activity. The epochs of the reversals of the polar field for the entire period 1870-1991 
were determined (Makarov and Sivaraman 1989, Makarov and Makarova 1996).

It was shown that the mean latitude of the filament band at the minimum of the cycle correlates with the maximum Wolf number in the succeeding cycle (Makarov and Mikhailutsa 1992). The dependence between them is given by

$$
W(N)=605-10.4\left\langle\Theta_{2, m}\right\rangle
$$

with the correlation coefficient 0.94 . The average latitude $\left\langle\Theta_{2, m}\right\rangle$ during 1996 was $39^{\circ}$, which corresponds to $W(23) \approx 200 \pm 20$.

Hence, the latitude-time distribution of the prominences is a rich source for the investigation of new global properties of solar activity. In the present report we give new results concerning long-term variations of the torsional oscillations of the Sun and quasi-periodic oscillations of the latitude of the zonal boundaries from an analysis of $\mathrm{H}_{\alpha}$ charts during the period 1915 to 1990 .

\section{Observational Data}

Continuous series of the latitude-time distribution of prominences were composed for 1880-1995 on the following bases:

- Meudon (d'Azambuja) charts - C.S.S.S. (1919-1964), Rot. 877-1486;

- Kodaikanal $\mathrm{H}_{\alpha}$ and CaII-K spectroheliograms (Makarov and Sivaraman 1989);

- Italian data (Ricco 1914, Bocchino 1935);

- Wolfer's Catalogue of prominences (Wolfer 1909);

- Kislovodsk Solar Observations (S.D.B. 1960-1995).

The Meudon charts of the filament distribution were completed by the daily observations of prominences. One can see that the prominence samples form global magnetic neutral lines which extend from $\mathrm{E}$ to $\mathrm{W}$ and form closed contours encircling a region of basically one polarity. Of course they contain some small weaker bipolar fields as well, especially at the photospheric level, but at 10,000 $\mathrm{km}$ above the photosphere the latter have lost their importance.

\section{Torsional Oscillations of the Sun Derived from the Distribution of Prominences}

Long-term variations of the differential rotation of the solar large-scale magnetic fields were investigated. $1024 \mathrm{H}_{\alpha}$ charts were used for the period 1915-1990. The method of field expansion in terms of Walsh functions was used. From 1915 to 1990, 7 bands of faster-than- and 7 bands of slower-than-average rotation were revealed. These bands drift towards the equator from $\Theta=45^{\circ}$ in 2.5 to 8 years. The width of the bands varies from 3 to 7 years and they are in anti-phase with the solar activity. The latitude length of the bands of torsional oscillations varies from $0.4 R_{\odot}$ to $1.3 R_{\odot}$ and they show a long-term variation of about 55 years. The poloidal component of velocity $V_{\Theta}$ varies from $2 \mathrm{~ms}^{-1}$ to $6 \mathrm{~ms}^{-1}$. 
The maximum velocity of the equatorial drift occurred in the period between 1935 and 1955 and it developed before the maximum activity of the strong 19th solar cycle. During the modern epoch from 1965 to 1985 the value $V_{\Theta}$ did not exceed $3 \mathrm{~ms}^{-1}$, but it shows now a tendency to increase. The bands of slowerthan-average rotation correspond to the evolution of magnetic activity towards the equator in the butterfly diagram (Makarov, Tlatov and Callebaut 1997).

\section{Oscillations of the Latitude of the Zonal Prominence Boundaries}

It was shown that in each hemisphere the zonal structure in the latitude-time distribution of prominences exist. These zones coincide with the boundaries of the large-scale rotation symmetric component of the magnetic field. $\mathrm{H}_{\alpha}$ charts make it possible to calculate the average per rotation of the latitudes of zonal boundaries $\Theta_{i}(t)$,

$$
\Theta_{i}(t)=\sum_{n} \Theta_{i, n}(t) / n
$$

where $i=1,3,5 \ldots$ denotes the boundary of $+/$ - polarity and $i=2,4,6 \ldots$ denotes the boundary of $-/+$ polarity (both starting from $\mathrm{N}$ pole). $\Theta_{i, n}=$ the latitude at the nth point of the longitude.

The latitude variations of the zonal boundaries during 1915-1989 consist of the poleward migration and quasi-periodic oscillations. The time-poleward migration depends on whether one-fold or three-fold field reversal occurs. In the case of cycles with one-fold field reversal the time of latitude boundary evolution is about 22 years. For the cycles with a three-fold reversal the zonal evolution time is about 8-12 years. The velocity of poleward migration of the latitude zones $V(N)$ is caused by the sunspot activity, as there is a correlation between $V(N)$ and the total sunspot area for the cycle (N-1), expressed in $10^{-3}$ area of the hemisphere, $V(N)=0.18 A^{2}+2.5 \mathrm{~ms}^{-1}$, where $A=\sum S p(N-1)$.

During 1915-1990 only one-fold polarity reversals were observed in the Southern hemisphere and the life-time of the boundaries was about 300 solar rotations $(\approx 22 \mathrm{yr})$. The latitude oscillations of the boundaries have a period of $16-20$ solar rotations (mean $\approx 18$ sol. rot, $4 \cdot 10^{7} s$ ), their horizontal velocity is about $5 \mathrm{~ms}^{-1}$ and the amplitude of the oscillations is about $5 \cdot 10^{7} \mathrm{~m}$. In the same period the sunspot area periods took about 10 solar rotations (Makarov, Petrova and Tavastsherna 1985).

In the Northern hemisphere one-fold and three-fold polarity reversals were observed and the life-time of the boundaries was about 170 solar rotations $(\approx$ $13 \mathrm{yr}$ ). The periods of latitude oscillations of boundaries are about 16-18 solar rotations. For fluctuations of sunspot total areas a period of about 6 solar rotations was found.

We conclude that the pattern of the latitude oscillations of the magnetic field with the period of about 18 solar rotations is not directly associated with sunspot activity, as it is observed at minimum activity too. The nature of those oscillations is not yet understood. However, we are now investigating any regularities or periodicities from the observational viewpoint. 


\section{Conclusions}

Of course each individual solar phenomenon has to be investigated fully. However, if one wants to obtain clues for the mechanism(s) of the global solar cycle, it seems important to emphasize the following:

- The need for investigating the long-term behaviour of each solar phenomenon.

- The need for correlating all the solar phenomena: the periods, the phases, the waxing and waning, the long-term variations, intensities, etc. Moreover this has to be done on a restricted scale too, e.g., torsional oscillations may be studied by Doppler-shifts, by $\mathrm{H}_{\alpha}$ charts, by the green coronal line, etc., in fact connecting the appearance of the same phenomenon at various depths.

Acknowledgments. This work has been done partly under the Collaboration Program Flemish Community-Russia. Two of us (V.I.M. and K.S.T) thank the Russian Foundation For Basic Research, Grant 96-02-16732 for financial support.

\section{References}

Bocchino G. 1935, Mem. Observ. Arcetri, 67, 7

Callebaut D.K. and Makarov V.I. Solar Phys., 141, 381

C.S.C.S - Cartes Synoptiques de la Chromosphere Solaire: 1919-1964, Meudon Makarov V.I. 1984, Solar Phys., 93, 393

Makarov V.I., Petrova N.S. and Tavastsherna K.S. 1985, Soln. Dann. Bull., 6, 69

Makarov V.I. and Sivaraman K.R. 1989, Solar Phys., 123, 367

Makarov V.I. and Mikhailutsa V.P. 1992, Solar Phys., 137, 385

Makarov V.I. 1994, Solar Phys., 150, 359

Makarov V.I. and Makarova V.V. 1996, Solar Phys., 163, 267

Makarov V.I., Tlatov A.G. and Callebaut D.K. 1997, Solar. Phys., 170, 373

Ricco A. 1914, Mem. Soc. Spectr. Ital., 33, 17

S.D.B. 1960-1995, Soln. Dann. Bull., Nos. 1-12

Wolfer A. 1909, Publ. Sternwarte Eitg. Polytechn., Zurich, B. 1-4, 1-57 